\title{
Una aproximación al concepto de Hecho Científico
}

\author{
Victor Patricio Díaz Narváez (victordiaz@uach.cl) Doctor en Ciencias Biológicas. Universidad Austral de Chile \\ Aracelis Calzadilla Nuñez. Médico Cirujano. Municipalidad de Coquimbo (Chile) \\ Hector López Salinas. Doctor en Ciencias Técnicas. Instituto Superior Politécnico José A. Echeverría (Cuba)
}

\begin{abstract}
To analyze the concept about scientific fact was the purpose of this work. This concept is a principal element to scientific hypothesis formulation. The properties and characteristics of this must observance were enumerated. Moreover, the concept studied of this work was compared with another analysis about some concept realized by other epistemologist and philosophic sciences. This concept is transversal for all patterns natural sciences and the properties and characteristics must observance.
\end{abstract}

Key words: scientific fact, scientific method, epistemology, philosophy of science

\section{Resumen}

El propósito de este trabajo es analizar el concepto de hecho científico. Este concepto es un elemento fundamental para la posterior formulación de hipótesis científica. Se enumeran las propiedades o características que estos deben cumplir para considerarlos como tales. Además, se compara el concepto tratado en este trabajo con el que han planteado algunos destacados pensadores en el campo de la epistemología y filosofía de las ciencias. Se concluye que este concepto es transversal a todos los tipos de ciencias naturales y, en todas ellas, las propiedades y características enunciadas deben cumplirse.

Palabras claves: hecho científico, método científico, epistemología, filosofía de la ciencia.

\section{Introducción}

Se plantea que el proceso del conocimiento transcurre por tres etapas fundamentales, las cuales pueden esquematizarse de la siguiente forma: a) la acumulación y elaboración de datos obtenidos mediante procesos empíricos; b) construcción y elaboración de la teoría sobre la base de la compilación y tratamiento de los datos empíricos obtenidos y c) la explicación de los datos empíricos conocidos, la deducción de predicciones a partir de los nuevos datos con la colaboración de la teoría elaborada, y la confirmación de la teoría por el material empírico (Fedoseev 1975, Eng 1987, García 1983, Pájaro 2002).

La división del proceso del conocimiento antes descrita es muy útil dado que nos permite dividir todos los problemas que le son inherentes y que pueden ser analizados, a su vez, en tres grupos: a) los de la etapa empírica del conocimiento que surgen durante la investigación; b) aquellos que se vinculan con la elaboración de teorías científicas (formulación de hipótesis, de leyes que emergen de acuerdo al nivel que se analice, etc.) y c) los problemas que emanan mediante el análisis de la relación entre el conocimiento teórico y el empírico (Fedoseev et al, 1975; Eng et al, 1987).

Como consecuencia, la división del proceso de la construcción del conocimiento nos permite focalizar nuestra atención en las diferentes etapas del proceso cognoscitivo pudiendo distinguir en él las particularidades y singularidades y precisar con mayor rigor y exactitud los problemas que son característicos o están relacionados con una u otra etapa del proceso cognoscitivo. 
Ponemos énfasis que la división antes planteada del proceso del conocimiento es sólo un esquema que intenta reflejar lo más adecuadamente posible el proceso cognoscitivo que transcurre en las ciencias. En la realidad práctica concreta, las etapas de este proceso del conocimiento no están nítidamente separadas entre sí, lo que implica que perfectamente la secuencia temporal puede no ajustarse al orden que hemos planteado.

La acumulación de datos empíricos puede transcurrir simultáneamente con la formulación de la hipótesis, y ambas se comparan constantemente con el material empírico. En el proceso de abordaje de una investigación empírica el investigador se guía por una determinada concepción del mundo, por las leyes fundamentales de las ciencias naturales y la metodología general del conocimiento científico, aunque no todos los científicos, de varias áreas del conocimiento, puedan necesariamente concordar con estas concepciones.

Se conoce también que en los diferentes períodos del desarrollo de una u otra ciencia, adquiere un fundamental significado la realización de diversos experimentos y la acumulación de materiales empíricos o la elaboración y perfeccionamiento de las representaciones teóricas o de las teorías en general (Fedoseev 1975, Díaz 2001a, Díaz 2001b). El objeto esencial de las investigaciones empíricas o de las investigaciones observacionales es la obtención de: "un grupo especial de juicios o proposiciones que señalan una relación o dato empírico, obtenido como resultado de la observación, del experimento, del resumen estadístico de un fenómeno o grupos de fenómenos reales" (Plasencia 1975). Este grupo especial es lo que denominamos hechos científicos.

En cualquier caso, toda investigación que tenga por objeto elaborar teorías sobre la base de hechos posibles de constituir sólidamente, requiere de la contrastación de dichas teorías con los hechos obtenidos. Al mismo tiempo, los propios hechos requieren de ser contrastados con algunas características, con el propósito de asegurar su solidez. Si estos no poseen ciertas características, el proceso del conocimiento, en términos de las etapas que le siguen a aquellas que les corresponde establecerlos, seguramente dichas etapas transcurrirán por caminos que podrían conducir a deducciones teóricas incorrectas o poco fundamentadas. Por tanto, la constitución de hechos científicos es la condición necesaria (aunque no suficiente), para las elaboraciones teóricas plausibles y, entre ellas, para la elaboración de la hipótesis científica.

\section{Características del Hecho Científico}

La etapa empírica del conocimiento científico incluye la acumulación y la elaboración primaria de los datos empíricos. El concepto fundamental y esencial en esta etapa es el de hecho científico. Este aparece en las ciencias naturales y la elaboración del método experimental. Existe una relación directamente proporcional entre la región de la ciencia que utiliza el método experimental y la significación que adquiere el análisis del este método. Este concepto sirve de punto de inicio del conocimiento científico, mientras que el conjunto de hechos se transforma en la base estructural del conocimiento científico-teórico, y estos se utilizan para confirmar o refutar las teorías científicas (Pájaro 2002).

Si consideramos que los hechos realizan ciertas funciones en la investigación científica es porque es posible atribuirles ciertas características o propiedades que permitan cumplir determinadas funciones. Estas propiedades son:

1. El hecho existe o se encuentra a disposición del investigador antes de la construcción de la teoría que pretende explicarlos. Esto se debe a que la teoría se construye para la explicación de los hechos. Por tanto, estos se constituyen en la premisa necesaria para la formulación de una teoría. En muchos casos, se requiere de una masa crítica de ellos antes de que pueda surgir una teoría que pueda unificar los hechos constituidos y sin aparente relación entre sí.

2. Los hechos se encuentran fuera de la teoría. Existe una "separación" del proceso de conocimiento en las etapas empírica y teórica, pero al mismo tiempo existe una correspondencia entre la teoría y los hechos. Dicha separación y correspondencia se produce en términos de que estos no pueden incluirse en la teoría que intenta explicarlos. Si 
fueran incluidos en la teoría no podrían servir como criterio de comprobación o refutación de la misma. Pero cuando la teoría es contrastada con los hechos y se va confirmando, la teoría como tal va incluyendo los hechos.

3. Los hechos son absolutamente auténticos. Como estos son empleados para la confirmación o refutación de la teoría, la correspondencia entre hecho y teoría se transforma en el criterio de admisibilidad de la teoría, debido a que ellos, una vez establecidos, no pueden someterse a la más mínima duda.

4. Los hechos son invariantes en relación con las diferentes teorías que se constituyen sobre su base. Como el hecho se encuentra fuera de la teoría y es absolutamente auténtico en relación con ella, no puede estar expuesto a ninguna transformación que transcurra en los niveles teóricos del conocimiento. Una teoría puede sustituir a otras, pero ellos se mantienen invariantes, lo cual obliga a determinada teoría, relacionada con la región de fenómenos ligada a determinados hechos, a orientarse por ellos. Como consecuencia, una determinada cantidad y tipo de hechos pueden constituirse en la base de teorías no sólo diferentes, sino hasta contradictorias.

5. Los hechos están vinculados con la teoría elaborada sobre su base. Si los hechos estuvieran totalmente desvinculados de la teoría, no podrían corregir su construcción ni servir de criterio de autenticidad. La elaboración, por tanto, de una teoría debe procurar el establecimiento de los vínculos y relaciones entre la teoría y los hechos.

Las propiedades de los hechos aquí descritos se determinan por la representación unilateral y abstracta del proceso del conocimiento, como proceso que se inicia por la selección de hechos y que posteriormente se mueve en el sentido de la construcción y elaboración de las teorías que los expliquen. No obstante, puede suceder que en el proceso del conocimiento real, estas propiedades pueden no corresponderse en general a los hechos científicos concretos o simplemente su correspondencia puede ser en menor grado. Esto puede deberse a que todo nuevo hecho de este tipo se descubre sobre las base de determinadas representaciones teóricas. Es conocido en la historia de la ciencia de casos en que los científicos no toman en consideración algunos descubrimientos que les permitieran que los mismos pudieran ser transformados en hechos, debido a la carencia o falta de desarrollo de determinadas concepciones teóricas. Si esto está planteado de este modo es posible señalar que las representaciones teóricas, en algunos casos, requieren de cierto desarrollo con anterioridad al descubrimiento del hecho científico.

Como las propiedades descritas no se manifiestan en estado puro en el descubrimiento de un hecho se requiere que, en el análisis metodológico del proceso del conocimiento, estemos obligados a realizar una abstracción de algunos aspectos del proceso cognoscitivo "orientándonos por la representación de este proceso según la cual las propiedades indicadas pueden atribuirse a los hechos" (Fedoseev 1975). El problema que surge entonces es tratar de explicar este concepto de manera tal que contenga las propiedades antes enumeradas.

\section{Una Aproximación al Concepto de Hecho Científico}

Al respecto, podemos señalar que se han planteado muchas "definiciones" del concepto de hecho: a) elemento de la realidad, b) elemento del conocimiento y c) resultado del reflejo sensorial.

Usualmente los hechos son denominados situaciones reales de los casos o fragmentos de la realidad. Bunge (1975) postula que es todo aquello que "pertenece a la realidad" y los divide en: acontecimientos, o modificaciones en el espacio-tiempo; procesos, o secuencia de acontecimientos ordenados en el tiempo; sistemas concretos o sustancias físicas; fenómenos, o el reflejo sensorial de los objetos y procesos reales en el sujeto cognoscente. Nosotros no podemos estar de acuerdo con la inclusión de la categoría fenómeno entre el número de hechos, puesto que estos no pertenecen a la realidad, y al ser tratados estos como tales nos conduciría a confundir el mundo real con el reflejo sensorial y no necesariamente entre estos dos últimos existe una coincidencia absoluta. Junto con los acontecimientos, procesos reales y objetos reales, a la realidad misma le pertenecen las propiedades y relaciones, como inherentes a los objetos y acontecimientos (Díaz y Calzadilla 2001). Existen también, junto a los hechos actuales, los posibles aún no determinados y que no contradicen las leyes de la naturaleza descubiertas hasta un momento dado. Esta concepción permite asignarle casi todas las propiedades antes enumeradas a los 
hechos, es decir, existen antes de la construcción de la teoría, se encuentran fuera de ella, son invariantes con relación a las diferentes teorías, pero no es aplicable la propiedad de poseer el carácter de verdaderos. Surgen también dos preguntas relacionadas con la concepción antes descrita acerca del hecho científico: a) ¿cómo poder asociar los acontecimientos reales, los objetos y procesos, con el conocimiento científico-teórico para que los primeros puedan servir de punto de partida en la elaboración de la teoría científica y de criterio de aceptabilidad, y que la teoría pueda lograr explicar los acontecimientos y procesos reales? ; b) ¿por qué se debe necesariamente considerar la realidad dividida en acontecimientos, objetos y procesos y no simplemente en otros tipos de fragmentos de dicha realidad tales como objetos y propiedades o en propiedades y relaciones?

La primera pregunta suele responderse sobre la base de que en el lenguaje de la ciencia se distingue una sección especial denominada el lenguaje empírico. Las proposiciones que elabora esta sección tienen la capacidad de describir los hechos de la realidad. La construcción de la teoría científica se apoya directamente en las proposiciones empíricas y no en los hechos, y la comprobación de la teoría se realiza sobre la base de las inferencias que se pueden obtener de ella, o con la ayuda de efectos empíricos, que posteriormente se comparan con los hechos. Pero en esta respuesta se mantiene el problema acerca de las posibilidades de poder realizar las correspondencias entre las proposiciones empíricas con los acontecimientos y procesos reales.

Tampoco es nítida una respuesta al segundo interrogante si admitimos como factible una comprensión abstracta del proceso del conocimiento, en donde los hechos existen con anterioridad a su elaboración científico-teórica y el conocimiento se inicia con la fijación de los hechos. El sujeto sólo puede fijar inicialmente esta división de la realidad. Las bases de la solución real a este problema fue planteado desde los tiempos de Kant y Marx, durante el cual fue enfatizado el papel activo y creador del sujeto cognoscente en su relación con la realidad. Dicho de otra manera: "El sujeto cognoscente aborda la realidad no como un espejo que fija toda influencia exterior, sino que refleja la realidad desde el punto de su actividad práctica y con ayuda de los medios intelectuales de elaboración de las influencias exteriores" (Fedoseev 1975). Como consecuencia, la división de la realidad en fragmentos sueltos es sólo el producto de las posibilidades limitadas que nos brindan los medios de conocimiento de la realidad, por los procedimientos de su esquematización, idealización y simplificación, más que por el hecho mismo de que la realidad esté dividida sólo en simples fragmentos.

En este sentido, el lenguaje es uno de los medios más importantes de esquematización de la realidad. El desarrollo de las investigaciones lógico-semánticas, iniciadas por Frege (1972) y Russel (1973), permitió elaborar un nuevo planteamiento del problema de la división de la realidad en fragmentos aislados. Estos trabajos mostraron que la distinción en la realidad de diferentes tipos de objetos está determinada por la cantidad de tipos de expresiones denominadoras aceptadas en el lenguaje (Ursul y Rodríguez 1982). Como consecuencia, si las expresiones individuales sólo son consideradas como designadoras, se deduce entonces que la realidad queda dividida por ese lenguaje solamente en algunos objetos. Por tanto, en este contexto, los hechos sólo pueden ser considerados sistemas concretos y las relaciones entre los objetos no tienen posibilidad de asignarle una expresión que las designe (Welti 1999). Por el contrario, si enriquecemos nuestro lenguaje con la introducción de expresiones que puedan designar propiedades y relaciones, entonces la realidad se divide en algo más que objetos aislados. En este caso, existirían tres tipos de diferentes sustancias y en el número de hechos estará contenido, además de las cosas, las propiedades y las relaciones.

Por lo tanto, la comprensión del hecho como fragmento de la realidad estará determinada, en un grado no menor, por la actividad del sujeto y, en particular, por el lenguaje. Este contexto, ayuda a tratar de responder la primera pregunta asociada a las relaciones de los hechos objetivos con el conocimiento teórico, sin considerar el valor objetivo que el hecho tiene por sí mismo.

Por otra parte, puede entenderse por hecho científico el elemento del reflejo sensorial (imagen sensorial) que es capaz de formarse en el hombre producto de la acción externa de la realidad. Bunge (1975) considera como tal no sólo a los procedimientos, procesos y cosas, sino también a los fenómenos de la conciencia. El análisis de los hechos científicos como elementos que son productos de nuestra capacidad sensorial es una característica del positivismo. El primer neopositivismo (Rodríguez 2001) proclamaba el dato sensorial como fundamento del 
conocimiento científico. Wittgenstein habla de los hechos como fragmentos de la realidad y los filósofos del Círculo de Viena, en general, proclamaban que el estudio de todo aquello que está atrás del dato sensorial (el problema de la división de la realidad) son pseudo problemas metafísicos (Melondo 2001). Por tanto, la única realidad es el dato sensorial y éste solamente tiene que ver con la ciencia, según el primer neopositivismo. Pero la comprensión de los hechos como lo sensorialmente dado ha tropezado con obstáculos insalvables (Osorio 1999, Díaz y Calzadilla 2002 ).

Si la realidad nos es dada mediante un reflejo del mundo exterior y sólo lo dado sensorialmente es la única realidad posible, tras lo cual nada existe, entonces resulta extremadamente difícil caracterizar a los hechos como verdaderos. La caracterización de estos como verdaderos, entendida sólo como lo dado sensorialmente, es inaplicable. Si en la comprensión (concepción) del hecho como fragmento de la realidad, todos los hechos eran "auténticos" (con las limitaciones ya planteadas anteriormente) en el sentido de que todos eran parte de la realidad misma y todos eran objeto de atención efectiva en la ciencia; bajo la concepción de que los hechos son como lo dado sensorialmente, arribamos a la conclusión de que estos se dividen en dos grandes grupos: aquellos que surgen como el producto de la acción exterior sobre nuestros órganos de los sentidos y los que aparecen como el resultado de la actividad de nuestra conciencia, entre las cuales podemos incluir a las alucinaciones, ilusiones y espejismos, entre otras. El problema que existe para la ciencia, bajo esta concepción, es que ambos son incluidos en la base del conocimiento científico. Este problema podría ser superado si existiera la posibilidad de separar un grupo de otro, pero ¿cómo distinguirlos si ambos son producto de los sensorialmente dado? La única forma de hacerlo es reconocer que lo sensorialmente dado es el resultado real y concreto de la acción mutua entre el sujeto y el mundo exterior (objetivo además) (Eliénkov 1977). Pero reconocer esto implica que el problema acerca del mundo exterior deja de ser un "pseudo problema".

Otro dilema que existe al tratar de explicar el problema que estudiamos está asociado con la imposibilidad del reconocimiento general de los hechos como lo sensorialmente dado. Las impresiones sensoriales son siempre impresiones de un sujeto determinado, por lo tanto, los hechos serán siempre de carácter subjetivo. De esta forma, el conjunto de hechos que puede tener un sujeto no tiene por qué ser coincidente con los de otro sujeto. Cada sujeto reconocerá en la ciencia sólo aquello que coincida con su propio conjunto de hechos. Esto nos lleva a pensar que cada individuo tendrá su propia ciencia, lo que nos conduce directamente al solipsismo (Rosental y Ludin 1983).

También existe el problema de su adecuada expresión en el lenguaje y, en términos más generales, el problema del vínculo de los hechos con el conocimiento científico-teórico. Todo parece indicar que este es un problema que no está completamente resuelto. Esto se debe a que, al expresar las impresiones sensoriales concretas mediante el lenguaje, es posible que se pierda el carácter individual e irrepetible de estas. Las impresiones sensoriales se elevan hasta llegar al concepto (Iliénkov 1977), pero estos fijan lo general inherente a toda una clase de estas impresiones y no conservan las características individuales que estas tienen. Como consecuencia, las proposiciones que expresan un determinado hecho no están limitadas a él, sino que pretenden expresar todo un conjunto de hechos que pueden ser representados por un concepto.

Debe ser objeto de atención la explicación del hecho como una determinada proposición del lenguaje de la ciencia. Formulaciones de esta índole han sido expresadas por varios autores (Schlick 1993, Popper 1980, Ayer 1978, Neurath 1970). De estas formulaciones surge la inquietud de conocer cuáles son los rasgos de las proposiciones que constituyen hechos científicos y en qué se diferencian de las demás proposiciones. Los hechos-proposiciones se diferencian de las demás proposiciones por su rol funcional en el sistema científico, por determinadas propiedades semánticas y por el modo de obtención (Fedoseev 1975).

En el sistema del conocimiento científico, estos hechos-proposiciones se constituyen en el punto de partida y son la información primaria sobre la cual se estructuran los conocimientos científicos. Por otra parte, son el fundamento de la autenticidad del conocimiento teórico de tal forma que su aplicabilidad será posible sólo si el conocimiento teórico coincide con ellos. Por tanto, las funciones que son atribuidas a los hechos-proposiciones son coincidentes o está muy cerca de las funciones atribuidas a las proposiciones de protocolo elaboradas por el Círculo de Viena. 
Pero, para obtener proposiciones que puedan cumplir la función de hecho en el lenguaje de la ciencia, el criterio funcional es del todo insuficiente por sí mismo debido a que estas funciones pueden atribuirse, en general, a cualquier proposición en un lenguaje científico (Fedoseev 1975). Esta es la razón por la cual al criterio funcional se le agrega el semántico. Así, en los hechos-proposiciones son considerados exclusivamente las proposiciones individuales; de esta forma, se arriba a la consideración de que la verdad de los hechos-proposiciones pueden fundamentarse fuera de la teoría y estas proposiciones desempeñan el rol de hechos científicos; en los hechosproposiciones sólo puede tener admisibilidad toda clase de conceptos que se refieran con exclusividad a las cosas y propiedades observadas. Al criterio semántico se le agrega, en algunas circunstancias, el llamado "pragmático", el cual indica el modo de cómo se obtienen los hechos-proposiciones. Estos, de acuerdo a este criterio, se obtienen directamente de los resultados experimentales, como protocolos de observación o como generalización de los protocolos de las observaciones.

De estas formulaciones emergen algunas dificultades: a) Resulta muy complicado definir cuáles son las proposiciones que efectivamente se constituyen en hechos científicos. Estos hechos-proposiciones no permiten arribar a una clara representación de qué es lo que existe tras las proposiciones. Tal afirmación se manifiesta en los ejemplos que proponen los partidarios de esta concepción, los cuales son muy diferentes en sus principios; b) Tal concepción nos conduce directamente al reconocimiento de la relatividad del concepto de hecho científico. Esto es así, toda vez que un hecho de este tipo no es tal por sí mismo, sino que este es un hecho de una determinada teoría, tomando en consideración que se formula, además, en el lenguaje de dicha teoría, todo lo cual nos lleva a deducir que los hechos-proposiciones comprendidos en una teoría son diferentes a los elaborados en otras teorías. Tal concepción nos conduce directamente a la renuncia de que los hechos científicos son invariantes, pues los aparatos conceptuales de dos (o más) teorías formularían hechos-proposiciones diferentes a causa que los aparatos conceptuales elaborados por estas lo son también y, c) Si el hecho se torna indispensable para la autenticidad de una teoría, "entonces con relación a la teoría deberá funcionar como un conocimiento absolutamente auténtico" (Fedoseev 1975). Tal aseveración está apoyada no sólo por su lógica interna, sino por la "extraña circunstancia" de que muchos científicos incluyen (o utilizan en forma práctica) entre los hechosproposiciones sólo aquellas proposiciones del sistema científico, las cuales se constituyen en verdades fuera de los límites de este sistema. Dentro de los límites de un determinado sistema científico las proposiciones que se constituyen en hechos no son sometidos a duda alguna, pero si estos hechos-proposiciones se transforman en proposiciones corrientes, pueden tornarse en verdaderas, así como falsas. Las consecuencias de tal situación son claras: entre los hechos pueden incluirse proposiciones falsas y aún, bajo las condiciones de correspondencia entre la teoría con sus respectivos hechos-proposiciones, no existe seguridad que una teoría sea auténtica. Por tanto, si los hechos son establecidos a partir de determinadas proposiciones, se anula la posibilidad de que los verdaderos hechos sirvan de base de la autenticidad de la teoría científica. Es decir, el hecho científico deja de cumplir su función fundamental.

Las dificultades de esta concepción no terminan con lo antes planteado. Si, además, imponemos a los hechosproposiciones un límite de que "sólo abarquen las proposiciones cuya verdad haya sido demostrada" (Fedoseev 1975), no será suficiente para eliminar el problema del establecimiento de la autenticidad de la teoría debido que se producirá otro problema, consistente en la verificación de las proposiciones que realiza la función de hechos. Esto nos conduce a que, en la verificación de estas proposiciones, tendremos la obligatoriedad de relacionarlas con el reflejo sensorial o con la realidad, introduciendo algún concepto para denominar sus elementos. Y esto, definitivamente, no es más que la función del concepto "hecho". Como consecuencia, la explicación de los hechos científicos simplemente como las determinadas proposiciones del lenguaje de la ciencia, no constituye una concepción que implique un "adelanto o avance metodológico" y no es posible sostenerla consecuentemente.

Las diferentes interpretaciones del concepto "hecho" podrían explicarse por los diferentes métodos de análisis del proceso del conocimiento científico. En las interpretaciones que apuntan y se limitan a establecer la relación mutua entre las diferentes partes del conocimiento científico es necesario y suficiente convertir en sistema de conocimiento aquellos elementos que actúan con un mayor grado de autenticidad en relación con otros. Dichos elementos pueden denominarse hechos-proposiciones o, incluso, pueden ser denominados con cualquier otro 
término. Pero si el conocimiento científico apunta hacia la realidad y, por tanto, se analiza desde el ángulo de las relaciones que se establecen entre el sistema de conocimientos con la realidad misma, el sistema del lenguaje de las ciencias se torna en un sistema estrecho que no nos posibilita establecer las relaciones antes nombradas y nos obliga a apoyarnos en lo sensorialmente dado o en los fragmentos de la realidad. Sólo en este caso los hechos surgirán como elementos del reflejo sensorial o de la realidad. En relación con las restantes proposiciones del lenguaje de la ciencia, los hechos-proposiciones realizan el mismo papel que tiene asignado lo sensorialmente dado y los hechos serán elementos del reflejo sensorial o de la realidad. En relación con las restantes proposiciones del lenguaje de la ciencia, los hechos-proposiciones desempeñan el mismo papel que tiene asignado lo sensorialmente dado en relación con el conocimiento racional o los elementos de la realidad en conexión con todo el conocimiento. De aquí se extrae que el concepto de hecho es relativo. Más, es relativo en el sentido de que el contenido de este concepto se determina por el modo de análisis del conocimiento y el papel de los enfoques y métodos generales empleados y, en última instancia, está relacionado con la concepción del mundo que el científico posea, pues es este último el que los determinará. Desde luego, esta determinación excede los objetivos de este trabajo.

La base empírica de las ciencias (el conocimiento de los hechos) se forma con ayuda de determinados métodos: observaciones, mediciones y experimentos, los cuales no discutiremos en el presente trabajo por razones de extensión y no de relación.

El concepto de hecho científico ha sido discutido, en las ideas precedentes, en relación con aquellos hechos que se establecen a partir de las ciencias particulares (Cadena 2001, Díaz y Calzadilla 2001) en general. Al respecto, y a modo de ejemplo, podemos observar que en una ciencia específica, como lo es la biología, existe concordancia en muchos de los científicos que pertenecen a esta área, en que los fenómenos vivientes deben ser pensados en términos de dos diferentes tipos de causación: las causas próximas o funcionales (que nos indica cómo es que algo ocurre); y las causas últimas o evolutivas (que nos indica por qué es que algo ocurre) (Mayr 1976, 1985, 1988, 1998, Jacob 1973, 1981, Sober 1993, Bunan 2000, Lewontin 1998, Willians y Nesse 1996, Magnus 2000, Futtuyma 2001). Pensada la biología de esta manera, es decir, dividida en dos grandes campos de indagación, se asocia a la biología funcional la labor de estudiar experimentalmente las causas próximas que nos explican el cómo de los fenómenos vitales; y la biología evolutiva, ocupada de reconstruir, por métodos comparativos e inferencias históricas, las causas últimas o remotas que nos explicarían el por qué de tales fenómenos (Caponi 2000, 2001). En esencia, estamos en presencia de una demarcación que es consecuencia de una distinción epistemológica que no tienen porqué observarse como contradictoria, sino más bien complementaria en una situación de alto grado de complejidad, por tanto las causas próximas pueden ser susceptibles de entrelazarse con las causas remotas. De hecho, las respuestas a ambas preguntas deben ser compatibles y, en principio, articulables las unas con las otras. "No existe nada parecido a una inconmensurabilidad entre biología funcional y biología evolutiva" (Caponi 2001), aunque los dominios de ocupación son decididamente distintos y dan respuestas diferentes, específicas para cada uno de estos dominios. El conjunto de argumentos antes planteados es válido para señalar que en los dominios de la ciencia biológica, aunque sean distintos entre sí, los hechos científicos se van configurando de tal forma que, a pesar de esta diferencia de dominios, los hechos científicos tienen características generales comunes para cualquiera de ellos.

Sin embargo, y generalizando aún más, es posible explorar si las propiedades de este concepto son válidas para fijar un mayor número de tipos de realidades en términos de hechos. Es decir, si tienen carácter más amplio y pueda ser útil para cualquier esfera de la realidad que se analiza, independientemente de su complejidad.

Si partimos sobre la base de que existe una conexión regular general de los fenómenos del universo y dicha "...conexión universal general de todos los fenómenos se refleja precisamente también en forma de conexión universal general de las distintas ramas y partes del conocimiento científico único del hombre sobre el mundo" (Kedrov 1974), quiere decir que hay que considerar seriamente la circunstancia de que las conexiones entre los fenómenos de la realidad pueden expresarse en forma directa o intermediada. Algunos fenómenos que están directamente relacionados entre sí, aparecen como relaciones de causa-efecto. Pero, además de los fenómenos 
conectados directamente uno con el otro y, correspondientemente, además de las conexiones entre las ciencias colindantes, que estudian objetos distintos, existen conexiones más alejadas, las cuales también tienen que reflejarse en el sistema de las ciencias, debido a que las ciencias más alejadas se conectan entre sí por eslabones intermedios.

Como consecuencia de lo anterior, si la conexión de los fenómenos como objetos de las distintas ciencias se establece en el curso de su propio desarrollo, la conexión entre las ciencias también se manifiesta en el curso de su desarrollo histórico, en otras palabras, en el curso del desarrollo del conocimiento humano (Díaz y Calzadilla 2001). La conexión de las distintas formas o fases del objeto, abarcado por las ciencias o sistemas de ciencias, dado en el tiempo también es desarrollo (cambio), que tiene una dirección determinada: de lo inferior a lo superior, lo cual implica un desarrollo progresivo, o su inverso, el desarrollo regresivo. Por lo tanto, los hechos científicos se van estableciendo en función del desarrollo del conocimiento humano en términos de hechos científicos de menor a mayor complejidad (de un número menor a un número mayor de conexiones involucradas en los hechos mismos).

Pero, la comprensión, por medio del conocimiento humano, de la conexión de las distintas formas o fases del objeto, es la comprensión de que dichas conexiones se producen objetivamente en el mundo estudiado, todo lo cual indica que en el propio mundo objetivo se producen tales conexiones (Iliénkov 1977, Díaz y Calzadilla 2002) y, por tanto, el objeto principal de las ciencias es estudiar las distintas formas de movimiento que se produce en este mundo objetivo. De aquí es que surge la necesidad de conocer las distintas formas de movimiento en la naturaleza, las conexiones y transiciones que se producen entre estos movimientos, en términos de desarrollo de la forma inferior (más simple) del movimiento hacia la forma superior (más compleja).

Por tanto, los hechos científicos están directamente relacionados con el tipo de movimiento que se estudia. Como consecuencia, existirán hechos científicos (y formas de establecerlos) "simples" y otros complejos, o cada vez más complejos. Esto obliga, a su vez, a poner atención a las condiciones y el carácter de las transiciones de una forma de movimiento en otra, además de las conexiones entre las ciencias y las transiciones de una ciencia en otra, lo cual no se examina en el presente trabajo.

Las ideas expuestas podrían ayudar a responder el porqué en algunas ciencias, tales como las experimentales, que se ocupan de regiones "estrechas" de la realidad (Química, Física, entre otras), la constitución y el establecimiento de hechos transcurren de una manera más "simple", mientras que en estudios, en las cuales el objeto de investigación implica la participación activa de varias ciencias simultáneamente para dar respuesta a un problema, la formulación de hechos es un problema en sí mismo y requiere de metodologías y enfoques nuevos para dichas constituciones y establecimientos y, consecuentemente, la solución del problema (Díaz y Calzadilla 2001).

Del conjunto de ideas precedentes, surge la siguiente pregunta: ¿Cambian las propiedades o características enunciadas anteriormente para el establecimiento de un hecho científico en la medida de que el número de conexiones y transiciones del objeto o los objetos analizados se tornan más complejos, dado que el objeto de atención se torna del mismo modo?

La respuesta a esta pregunta dependerá de los postulados iniciales que se consideren. Si asumimos los de los positivistas, neopositivistas o cualquiera de sus variantes (a modo de ejemplo), los cuales plantean en general que "el mundo exterior debe considerarse como una acumulación infinita, como un simple montón de hechos 'elementales' absolutamente independientes unos de otros, y la 'correcta definición de cada uno de ellos deben ser independiente en absoluto de la definición de cualquier otro hecho" (Iliénkov 1977), entonces el "estudio científico del mundo" se limitará a la unión puramente formal y verbal de un cúmulo de hechos sueltos, mediante su puesta en un mismo término bajo un mismo "común". Este "común", interpretado como "significación del término o signo", siempre resultará algo completamente arbitrario o "establecido de antemano por acuerdo", es decir, "convencional" (Díaz y Calzadilla 2002). Como consecuencia, lo "común" (la unidad y la identidad), como único resultado de la elaboración "científicamente lógica" de los "hechos elementales", no es el resultado de la realidad, sino de la "significación del término", convencionalmente establecido de antemano (Iliénkov 1977). De este modo, 
las características atribuidas a los hechos científicos enunciados en este trabajo no se cumplirían y no tiene la mayor relevancia si los objetos son simples o complejos en sí mismos.

Las consecuencias que tienen estos postulados para las ciencias no constituyen una cuestión menor. Bajo esta concepción, los objetos simples, menos aún los complejos, no podrían abarcarse y, al mismo tiempo, la filosofía (algunos la denominan metafísica) no se constituiría como una herramienta más del conocimiento. De esta forma, sería imposible tratar de explicar los fenómenos sociales (fenómenos que requieren de un estudio específico dada su naturaleza), debido a que (expresado aún en forma simplista) estos son el producto de complejas interacciones de numerosos factores en donde el peso específico de algunos de ellos puede mantenerse relativamente constante en el tiempo, mientras que otros pueden variar de una forma suave o brusca en él.

Los estudios realizados para proponer métodos y enfoques con el objeto de tratar de reflejar de alguna manera la variedad y complejidad de las conexiones que existen dentro y entre los objetos de estudio (Arnold y Osorio 1998, Ricci 1999, Bar 2000, Díaz y Calzadilla 2002, Andrade 2002, Escobar 2002), permiten confirmar el carácter de las conexiones. De otra manera, no sería de interés de los científicos poner atención a la formulación de nuevos métodos y enfoques que permitan elaborar hechos científicos en su región del conocimiento.

El problema que persiste es saber si estas conexiones se corresponden con la realidad y si es posible conocerlas mediante el establecimiento de hechos científicos. En otras palabras, si la realidad misma es compleja en sí misma. Pero, a modo de ejemplo también, si tomamos como cierto el postulado de Maturana (1977), Maturana y Varela (1994) y Ruiz (1996) de que el conocimiento opera como un sistema determinado sólo desde el interior mediante sus propias estructuras y que esto trae como consecuencia que el observador no puede dar explicaciones de la realidad, si no solamente de las que han sido producidas por sus propias operaciones y que ocasionan un cambio estructural determinado en su estructura, existiendo tantas explicaciones como observadores participen en ellas, entonces no tendremos la más mínima oportunidad de abarcar la realidad y, mucho menos, de establecer hechos simples o complejos, pues estaremos impedidos de saber si una de esas tantas explicaciones se corresponde con una realidad común (y objetiva, además) y si el proceso de establecimiento del propio hecho científico vaya incidiendo sobre un observador en forma distinta con respecto a otro, en virtud de que en cada uno de ellos transcurren operaciones singulares que ocasionan cambios estructurales singulares de su propia singular estructura. Esta concepción resulta muy parecida al agnosticismo (entendiendo por tal aquella tendencia filosófica que admite la existencia de la realidad objetiva, pero que, a su vez, señala la imposibilidad de conocer tal realidad) (Rosental e Ludin 1983) con claros matices de reduccionismo biológico (Díaz y Calzadilla 2001).

\section{Conclusiones}

Retomando la pregunta precedente es posible señalar que: si tomamos como cierto que el conocimiento humano progresa en el sentido de ir reconociendo o apropiándose de las relaciones y las interconexiones entre los múltiples objetos (múltiples no sólo en número, sino también en complejidad); que el propio objeto va transformándose en el tiempo, en virtud del carácter de la apropiación de dichas complejidades y que las mismas tienen fuentes naturales (existen en la propia naturaleza); y que en la naturaleza existen distintos tipos de movimiento, entonces podemos señalar que los hechos científicos, en cualquier esfera particular de la ciencia y en el propio sistema de las ciencias, deben cumplir con las propiedades y características ya señaladas y que el establecimiento de los mismos puede ser en sí mismo de mayor o menor complejidad o puede requerir de métodos y enfoques nuevos cada vez más complejos, lo cual resulta como una acción necesaria pero jamás suficiente para abarcar la infinita complejidad de la naturaleza misma. La complejidad de la determinación del hecho estará en correspondencia directa (o más o menos directa) con la complejidad misma alcanzada en la esfera analizada.

\section{Agradecimientos}

Entrego mi reconocimiento a los Profesores Ricardo Oyarzún (Ms) y Juan Carlos Skewes (Ph.D) de los Institutos de Salud Pública y Ciencias Sociales de la Universidad Austral de Chile, respectivamente; así como al Profesor Sergio H. Menna (Ph.D) de la Facultad de Filosofía y Humanidades de la Universidad Nacional de Córdoba, Argentina, por los 
comentarios y sugerencias realizados a este trabajo. Muchos de estos comentarios y sugerencias fueron incluidos en el presente texto; otros no lo fueron a causas de diferencias de enfoques, a pesar de que estos estaban respaldados por argumentos sólidos desde el punto de vista teórico. Dichas diferencias son legítimas en el campo de las ideas que se discuten en este trabajo.

\section{Bibliografía}

ANDRADE, E; PACHANO, E; PEREIRA, L; TORRES, A. 2002. "El Paradigma Complejo". Revista Cinta de Moebio. Facultad de Ciencias Sociales. Universidad de Chile. № 14, Septiembre.

ARNOLD, M; OSORIO, F. 1998. "Introducción a los Conceptos Básicos de la Teoría General de los Sistemas". Revista Cinta de Moebio. Facultad de Ciencias Sociales. Universidad de Chile. № 3, Abril.

AYER, A.J. 1978. Positivismo Lógico. Editorial Fondo de Cultura Económica. México.

BAR, A. 2000. "Una Tipología de Métodos generales desde una Perspectiva Sistémica". Revista Cinta de Moebio. Facultad de Ciencias Sociales. Universidad de Chile. № 7, Marzo.

BUNGE, M. 1975. La Investigación Científica. Editorial Ciencias Sociales. La Habana.

BURIAN, R. 2000. The impact of genetics on biological theory. Résumés du Coloque: 1900, Redécouverte des lois de Mendel. Académie des Sciences. Paris.

CADENA, R. 2001.La Filosofía de la Ciencia. http://www.rcadena.com/ensayos/filcienc.htm

CAPONI, G. 2000. "Cómo y por qué de lo viviente". Ludus Vitales 7 (14): 67-102.

CAPONI, G. 2001. "Biología Funcional vs. Biología Evolutiva". Episteme (12): 23-46.

DÍAZ, V.P. 2001a. "Marco Teórico-Conceptual para un Sistema de Investigaciones Científicas. I. Papel de la Teoría y el Enfoque Sistémico en la Ciencia Contemporánea". Revista Universidades. Universidad Autónoma de México. 5 (20): 35-43.

DÍAZ, V.P. 2001b. "Marco Teórico-Conceptual para un Sistema de Investigaciones Científicas. II. El Concepto de Potencial Científico". Revista Universidades. Universidad Autónoma de México. 5 (20): 43-51.

DÍAZ, V. P; CALZADILLA, A. 2001a. "Papel de las Investigaciones Interdisciplinarias en el desarrollo del conocimiento. Una aproximación teórica". Revista Ensayo y Error. Universidad Simón Rodríguez. Caracas. 10(21): 97-110.

DÍAZ, V. P; CALZADILLA, A. 2001b. "El Reduccionismo, Antirreduccionismo y los Métodos Generales del Conocimiento Científico". Revista Cinta de Moebio. Facultad de Ciencias Sociales. Universidad de Chile. № 11, Septiembre.

DÍAZ, V. P; CALZADILLA, A. 2002. "Descartes y Espinosa en la filosofía de la ciencia contemporánea". Revista Ensayo y Error. Universidad Simón Rodríguez. Caracas. 11(22): 41-63.

ENG, A; CANTERO, M; VERGARA, D. 1980. Metodología de la Investigación. Departamento de Investigaciones Sociales. Editorial del Centro de Información del MICONS. La Habana.

ESCOLAR, C. 2002. "La teoría menor, el Tiempo Histórico y la Práctica Simbólica Compartida". Revista Cinta de Moebio. Facultad de Ciencias Sociales. Universidad de Chile. № 15, Diciembre.

FEDOSEEV, P.N; RODRígUEZ, M; RUZAVIN, G. 1975. Metodología del Conocimiento Científico. Editorial Ciencias Sociales. La Habana.

FREGE, G. 1972. "Sobre Sentido y Denotación". En: Lógica y Semántica. Ediciones Universitarias. Valparaíso.

FUTTUYMA, D.J. 2001. "El conocimiento científico". Elementos (8) 41: 1-14.

GARCÍA, H. 1983. Manual de Investigación Aplicada en Servicios Sociales y de Salud. Ediciones Copilco, S.A. México D.F. 
ILIÉNKOV, E.V. 1977. Lógica Dialéctica. Editorial Progreso. Moscú.

JACOB, F. 1973. La lógica de lo viviente. Laia. Barcelona.

JACOB, F. 1981. Le jeu des posibles. Fayard. Paris.

KEDROV, B.M. 1974. Clasificación de las Ciencias. Tomo I. Editorial Progreso. Moscú.

LEWONTIN, R. 1998. "Las base del conflicto en biología". En: Historia y explicación en biología. Martinez, S; Barahona, A (eds). México.

MAGNUS, D. 2000. "Down the primrose path: competing epistemologies in early XX century biology". En: Biology and Epistemology. Creath, R; Mainchistein, J (eds). Cambridge University Press. Cambridge.

MATURANA, H. 1977. El sentido de lo humano. Dolmen Ediciones S.A. Santiago de Chile.

MATURANA, H; VARELA, F. 1994. De máquinas y seres vivos. Autopoiesis: La organización de lo vivo. Quinta edición. Editorial Universitaria. Santiago de Chile.

MAYR, E. 1976. Evolution and the diversity of life. Cambridge; Harvard University Press.

MAYR, E. 1985. "How biology differs from the Physical Sciences". En: Evolution at a Crossroads. Depew, D.S.;

Weber, B (eds). Cambridge; MIT Press.

MAYR, E. 1988. Toward a new philosophy of biology. Cambridge. Harvard.

MAYR, E. 1998. O desenvolvimento do pensamento biológico. Brasilia. Universidade de Brasilia.

NEURATH, O. 1970. Fundations of the Social Sciences. University of Chicago Press. Chicago.

MELONDO, T. 2001. Para "salvar" la filosofía primera frente a sus versiones "débiles". El positivismo lógico, el primer Wittgenstein, Carnap, la hermenéutica de Gadamer.

http:/www.geocities.com/Athens/Forum/5284/melondo1.html

OSORIO, F. 1999. ¿Qué es el Positivismo Lógico? http://rehue.csociales.uchile.cl/antropologia/positivismo.htm

PÁJARO, D. 2002. "La Formulación de la Hipótesis". Revista Cinta de Moebio. Facultad de Ciencias Sociales.

Universidad de Chile. № 15, Diciembre.

POPPER, K. 1980. Conjeturas y Refutaciones. Editorial Paidos. Barcelona.

RICCI, R. 1999. "Acerca de una Epistemología Integradora". Revista Cinta de Moebio. Facultad de Ciencias Sociales. Universidad de Chile. № 5, Abril.

RODRÍGUEZ, J. 2001. Empirismo (o Positivismo) Lógico - Neopositivismo.

http//www2.alcala.es/estudios_de_organización/epistemologia/empirismo-logico.htm

ROSENTAL, M; LUDIN, P. 1983. Diccionario Filosófico. Editora Política. La Habana.

RUIZ, A.B. 1996. "The constributions of Humberto Maturana to the sciences of complexity and psychology". Constructivist Psychology 9 (4): 283-302.

RUSSELL, B. 1973. Historia de la Filosofía Occidental, la Sabiduría de Occidente. Editorial Aguilar. Madrid.

SCHLICK, M. 1993. "Positivism and Realism". En: The Philosophy of Science. The MIT Press. Cambridge.

SOBER, E. 1993. Philosophy of Biology. Oxford University Press.

URSUL, A; RODRÍGUEZ, M. 1982. La Dialéctica y los Métodos Científicos Generales de Investigación. Editorial de Ciencias Sociales. La Habana.

WELTI, M. 1999. La transición de Wittgenstein del Tractatus, al último Wittgenstein.

Http//www.info.pue.udlap/bajocenizas/witt.html 
WILLIANS, G; NESSE, E. 1996. Evolution and Healing: the new sciences of Darwinian medicine. Phoenix. New York.

Recibido el 18 Nov 2004 